Sometimes the successive escape of several stones from the kidney gives rise to several consecutive attacks of stone in the bladder. $D$. Lastly, if a fragment is left after an operation, it may serve as a nucleus for another stone, as in case 16; as a guard against such retention of fragments after litholapaxy, the bladder should always be washed out with the evacuator one or more times after the operation, before discharging the patient, which can readily be done without ether; in using the pump, the sacculated character of many bladders should be remembcred, and a careful search should be made, lest a fragment be caught and held in some side pocket; the orifice of the evacuating tube should be turned successively toward each part of the cavity, in order to dislodge with the current any such fragment.-Boston Med. and Surg. Jour., Dec. 2 and 9, 1886.

\title{
ULCERS, TUMORS.
}

1. The Cure of Large Ulcers of the Leg by the Carbolized Spray, By Gilles Dl: LI Tourfitre (Paris). This paper presents in detail three cases of stubborn ulcers of the leg in inmates of the Infirmeric des Incurables connected with the service of Charcot. The general condition of the patients was of the worst description. The first was an extremely debilitated and emaciated subject of asthma conjoined with chronic bronchitis, 69 years old, presenting an enormous ulcer covering the whole right leg, from the malleoli to within an inch of the tuberosity of the tibia. The carbolic spray for an hour and a half morning and evcning, with intermediate dressing with borated vaseline, caused a complete cure in less than a month. The second was an ulcer, 8 centimeters high, covering the lower half of the leg in a patient $8 z$ years of age, feeble and senile and the subject of chronic bronchitis. Carbolized spray for two hours twice a day with borated vaseline dressing in the intervals, secured a complete cure in about six weeks. The third case occurred in a syphilitic subject, with mitral insufficiency, tertiary syphilis and chronic bronchitis, aged 59. with a vast ulcer, 22 centimeters long, enveloping the whole leg. Carbolic spray applied during the next six months had brought the ulcer down to about the size of a six sous piece when the patient died by 
his own hand. As the result of his observations, the author concludes: a. The method of carbolic spray repeated dai,y for an hour and a half, moming and evening, better than any other method leads to a rapid cure of large varicose ulcers. $b$. In the early part of the treatment, the pains seated in the ulcer disappear. In the three cases ob. served, no erythema ever appeared at the margin of the wound, nor did, the patient ever void the black urine indicating carbolic poisoning. $c$. A state of debility or senility of the patient does not in any way contraindicate the employment of the method, which, on the contrary, by the local stimulation, which it determines, seems formally indicated in this particular case. $d$. The solutions used are the stronger, as the ulcer is the more atonic; solutions less than 1 to 50 should be rejected and even the greater strength, $1 / 30,1 / 30$, or even $1 / 10$ could be used. In the interval between the applications of the spray. the dressing of borate of soda and vaseline, I to to, will be found useful.-Revuc de chirurgic, July, 1886.

\section{The Treatment of Anal Fistula Associated with} Phthisis. By E. E. Glover M. D., (Terte Haute, Ind:). This paper is a résumé of the opinions of a considerable number of American surgeons obtained in answer to a series of questions propounded by the autior, and reflect, he believes, the prevalent opinions of the profession in the United States. 1. Operative interference is advised and practiced with benefit to the patient, excepting (a) where the cough is constant, unless this be first allayed; $(b)$ where the pulmonary disease is either rapidly advancing or is far advanced; or $(c)$ where the reparative fowtrs of the patient are so low that they are evidently unequa to the task of healing the wound. 2. Although it is proper to operate during any season, preference should be given to pleasant weather, such as will allow the patient to be in the open air. 3. Where the tissue surrounding the fistulous track is supposed to be tubercular, some advise its removal by the knife or sharp.spoon. 4. The wounds heal in nearly every case in which an operntion is justifiable. There shculd be as little interference with the sphincter muscles as possible. 5. The suppression of the discharge is supposed to be positively beneficial. It is recommended by some that where the discharge is supposed to have a beneficial derivative effect, a seton be inserted in the 
arm or other eligible part before operating on the fistula. 6 . It is believed that a successful operation tends to retard the progress of the disease and to prolong the life of the patient.-Jour. Am. Med. Ass'n., Nov. 20. I886.

J. E. Pilcingr (U. S. Armyi

III. Cases of Echinococcus in Man. By Dr. A. E. Fick (Richmond, Cape-Colony). No cases of echioococcus haviog been reported from Africa (with the exception of those described by Bitharz), the author believes his to command a certain interest.

From July, 1879 , to January. 1886 , he saw nine cases, only five of which, however, were treated by operations, and the diagnosis thus verified.

From his experience he draws the following conclusions :

1. The presence of albumen in the fluid of a cystic tumor does oot preclude echinococcus.

2. Tapping is to be entirely discarded as a mode of treatment, because the dangers cornected with it are coosiderable, and because the radical operation, in case it should subsequently become necessary, is thereby rendered much more difficult and dangerous.

3. Aspiration for diagnostic purposes should be limited to a small amount of fluid, at most to $50 \mathrm{cc}$.

4. The dangers connected with the radical operation of oon-suppurative cysts consist principally in failure to remove parts of the echinococcus, even wheo the abdomeo is opened. Eotrance of the fluid into the abdominal cavity is comparatively harmless. The iocision should, therefore, if possible, be made sufficiently large to admit the whole hand in to the cyst, in order to completely empty it.

5. Operation should consist in incision (a) whenever the cyst is small and superficially situated, so that the operation may be easily conpleted and the recovery rapid; (b) if the presence of the cyst interfere with the patient's occupation. Not easily operable cysts causing no special inconvenience, should not be interfered with, since it ap- 
pears to be not uncommon that echinococci spontaneously perish, break down, and shrink.

The cases are in short as follows:

I. Boy of 12 years, presenting an hepatic tumor reaching to the crista ilii, and causing moderate inconvenience. Diagnosis confirmed by another physician. Some years later patient was seen again, when the tumor had entirely disappeared.

II. Girl, xt. I2, colored; very large tumor of liver, taking up the whole right side. Hydatid cyst diagnosed, and unfavorable prognosis made. Patient at present healthy and strong, with no trace of tumor.

III. Strong man, 40 years, presenting a tumor of liver, troublesome at times. Echinococcus diagnosed, but operation not advised, as the tumor did not increase in size. (None ot these cases were operated upon).

IV. Elderly farmer, seen only once; presented a fistula under right costal margin, which secreted pus and admitted a catheter for 10 or 15 cm. Diagnosis: Remnant of hydatid cyst.

V. Young man, æt. 16 , suddenly attacked with pain in right lumbar region; subsequently pains in bladder and hip. Urine contains small echinococcus-cysts, the size of a pea.

Int ision (Dr. Hohmann) parallel to margin of quadratus lumborum muscle; numbers of cysts evacuated. Recovery said to have lasted four months. At present healthy condition.

VI. Child, at. 4. Tumor of left lobe of liver, fluctuating. Diagnostic aspiration vented jo $\mathrm{cc}$. of non-albuminous clear liquid, in which no scolices were found. Temperature next day $40^{\circ} \mathrm{C}$, attributed to a dulness in apex of right lung, reaching to second rib. As blood was expectorated from time to time, the diagnosis of pulmonary echinococcus was made. Treatment with tincture. of Kamala, $3^{\circ}$ drops, three times a day, an operation not being permitted.

VII. Man, æt. 29. Had been tapped for a tumor occupying the left hypochondriac region, reaching to within $2 \mathrm{~cm}$. of the iliac crest. Cardiac dulness continuous with that of tumor. Incision through upper part of left rectus abdominal muscle (where previously tapped). 
The spleen only presenting here, another incision at right angles was added. Cyst found and stitched to abdominal wound; suture of rest of wound. Incision of sac; irrigation with carbolic acid $2 \%$. Counter-opening made for insertion of drainage-tube into sac. Reactionary temperature, $38.6^{\circ}$ Abdo minal incised wounds healed by primary intention. Contents of sac, however. became putrid. On gth day $40.0^{\circ} \mathrm{C}$. Sac cleaned out. but fever continued for four weeks, and recurred when patient got up. After six weeks recovered, but fistula remained. One year later, in endeavoring to close the fistula, communication was accidently established with colon, but finally total recovery was achieved. At present patient suffering from catarth of apex of lung.

VIII. Girl, xet. 21, first noticed symptoms of disease four or five years previously. Scoliosis. Hepatic dulness $23.5 \mathrm{~cm}$. in the line of right papilla. Diagnostic aspiration reveals a clear, albuminous fluid. No scolices. Reactionary rise of temperature, and pain with tympanites for six days. Operation September, 1885. Incision. Suture of liver to edges of wound; partial evacuation of cyst; further row of sutures; incision into cyst through covering layer of liver-tissue: hæmorrhage; third row of sutures. Dressing of iodoformized gauze, dry sublimated cotton and borated bandages. On the following days vomiting. Temperature, $38.5^{\circ} \mathrm{C}$. Pulse 220 . Cyanosed lips. Antipyrin. After two weeks fever had risen to $40.0^{\circ} \mathrm{C}$. No putrefaction of cyst, the parts of which came away for the next five weeks. After third month only a fistula remained, which is expected to close in another month. Patient doing well.

IX. Girl, at. 22, presenting an hepatic tumor, of moderate size. Aspiration of $500 \mathrm{cc}$. of clear albuminous liquid, containing scolices. On following days abdomen painful, sumewhat distended. Temperature $39.5^{\circ} \mathrm{C}$. In three weeks dismissed improved. After four months patient was seen again, in bad general condition, and complaining of severe pain and some fever. Diagnostic aspiration produced pure pus. Reactionary temperature $40.0^{\circ} \mathrm{C}$. Pulse 120 . Incision between ninth and tenth rib. No pus found. 
Wound closed again with sutures, healed by first intention. After one week fever returned, but subsided after some further weeks, and patient began to improve in health. Was dismissed after one month. -Deulsche Zeitschr. f. Chirurg., Vols. 24, 3 and 4. Heft 25, August, 1886.

W. WV. VAN ARSDAlE (New Yort 1. 